\title{
Efficacy and safety of Qinzhuliangxue decoction for treating atopic eczema: a randomized controlled trial
}

\author{
Tian Ma ${ }^{1 \#}$, Yuanyuan Chai ${ }^{1,2 \#}, \mathrm{Su} \mathrm{Li}^{1}$, Xiaoying $\mathrm{Sun}^{3}$, Yifei Wang ${ }^{1,3}$, Rong Xu ${ }^{1}$, Jie Chen ${ }^{1}$, Mi Zhou ${ }^{1}$, \\ Min Zhou ${ }^{1}$, Bin $\mathrm{Li}^{1,3}$, Wenbin $\mathrm{Xu}^{1}$, Xin $\mathrm{Li}^{1,3}$ \\ ${ }^{1}$ Department of Dermatology, Yueyang Hospital of Integrated Traditional Chinese and Western Medicine, Shanghai University of Traditional \\ Chinese Medicine, Shanghai 200437, China; ${ }^{2}$ Department of Surgery of Traditional Chinese Medicine, Xiangshan Traditional Chinese Medicine \\ Hospital, Shanghai 200020, China; ${ }^{3}$ Institute of Dermatology, Shanghai Academy of Traditional Chinese Medicine, Shanghai 201203, China \\ Contributions: (I) Conception and design: W Xu, X Li; (II) Administrative support: B Li; (III) Provision of study materials or patients: S Li, X Sun, Y \\ Wang, M Zhou; (IV) Collection and assembly of data: R Xu, J Chen, M Zhou; (V) Data analysis and interpretation: Y Chai, T Ma; (VI) Manuscript \\ writing: All authors; (VII) Final approval of manuscript: All authors. \\ \#These authors contributed equally to this work. \\ Correspondence to: Xin Li; Wenbin Xu. Department of Dermatology, Yueyang Hospital of Integrated Traditional Chinese and Western Medicine, \\ Shanghai University of Traditional Chinese Medicine, Shanghai 200437, China; Institute of Dermatology, Shanghai Academy of Traditional Chinese \\ Medicine, Shanghai 201203, China. Email: 13661956326@163.com; xuwenbin@shyueyanghospital.com.
}

Background: Atopic eczema is the most common type of skin disorder in both children and adults. It
is characterized by erythema, pruritus, papules, xeransis, and lichenification. Qinzhuliangxue decoction
(QZLXD), a Chinese herbal medicine (CHM) prepared with several ingredients that are used to treat
eczema, was formulated according to the traditional Chinese medicine (TCM) theory. This study aimed to
investigate the efficacy and safety of QZLXD administration for treating atopic eczema compared to those of
Runzaozhiyang capsules (RZZYC).

Methods: A total of 176 patients were enrolled at the Shanghai Yueyang Hospital and were randomly assigned to the QZLXD treatment group $(n=82)$ or the RZZYC control group $(n=86)$. The differences in Eczema Area and Severity Index (EASI), Dermatology Life Quality Index, itching score, recurrence rate, and adverse events (AEs) were compared between the groups.

Results: The EASI score $\left(\chi^{2}=14.181, \mathrm{P}=0.003\right)$, recurrence rate $\left(\chi^{2}=7.398, \mathrm{P}=0.007\right)$, and itching score $(\mathrm{F}=-3.427, \mathrm{P}=0.001)$ were lower in the QZLXD group than in the RZZYC group. Incidence of AEs was similar between the RZZYC and QZLXD groups ( $\mathrm{P}=0.434)$.

Conclusions: QZLXD is recommended for the treatment of subacute atopic eczema because QZLXD showed good efficiency with low recurrence rate and tolerable AEs.

Keywords: Atopic eczema; clinical research; Qinzhuliangxue decoction (QZLXD)

Submitted Oct 09, 2019. Accepted for publication Feb 14, 2020.

doi: 10.21037/apm.2020.04.17

View this article at: http://dx.doi.org/10.21037/apm.2020.04.17

\section{Introduction}

Eczema refers to a group of skin disorders, including atopic eczema, contact eczema, and stasis dermatitis, of which atopic eczema is the most common type (more than $50 \%$ cases) (1). It develops in both children and adults and is characterized by erythema, pruritus, papules, xeransis, and lichenification (2). The incidence of eczema is $4.6 \%$ in China (3), whereas in the United States, morbidity due to eczema among adults is $7.2 \%$ (4), resulting in annual treatment costs of $\$ 5.297$ billion USD (in 2015) (5).

Currently, there is no cure for eczema, and treatment involves the use of basic emollient formulations; topical anti-inflammatory, photo, and antipruritic therapies; 
Table 1 Composition of RZZYC

\begin{tabular}{llll}
\hline Chinese Pinyin & Pharmaceutical name & Genus & Species \\
\hline He Shouwu & Polygonum multijiorum Thunb. & Polygonum & Thunb \\
Zhi Heshouwu & Fallopia multiflora (Thunb.) Harald. & Fallopia PRAEPARATA & Harald \\
Sheng Dihuang & Rehmannia glutinosa Libosch. & Rehmannia & Libosch \\
Sang Ye & Morus alba L. & Morus & Alba \\
Ku Shen & Sophora flavescens Ait. & Sophora & Ait \\
Hong Huoma & Bredia tuberculata (Guill.) Diels & Bredia & Diels \\
\hline
\end{tabular}

RZZYC, Runzaozhiyang capsule.

Table 2 Composition of QZLXD

\begin{tabular}{llll}
\hline Chinese Pinyin & Pharmaceutical name & Genus & Species \\
\hline Fang Feng & Saposhnikovia divaricata (Turcz) Schischk. & Saposhnikovia & Schischk \\
Gan Cao & Glycyrrhiza uralensis Fisch. & Glycyrrhiza & Fisch \\
Huang Qin & Scutellaria baicalensis Georgi & Scutellaria & Georgi \\
Mu Danpi & Paeonia suffruticosa Andr. & Paeonia & Andr \\
Yi Yiren & Coix lacryma-jobi L. var. mayuen (Roman) Stapf & Coix & Stapf \\
Mu Li & Ostrea gigas Thimberg & Ostrea & Thimberg \\
Zhen Zhumu & Hyriopsis cumingii (Lea) & Hyriopsis & Cumingii \\
Ci Shi & Magnetite & Magnetitum & Mineral \\
Zi Cao & Arnebia euchroma (Royle) Johnst & Arnebia & Johnst \\
\hline
\end{tabular}

QZLXD, Qinzhuliangxue decoction.

frequent cleaning; and dietary interventions, which are prescribed based on the individual's clinical condition during the long course of treatment (6). Additionally, western treatment strategies for mild-to-moderate atopic dermatitis include topical administration of corticosteroids, which have limited efficacy toward moderate-to-severe atopic dermatitis (7). Besides, systemic ciclosporin and corticosteroids are restricted for long-term use because of their limited efficacy and potential toxic and side effects, such as allergies, skin thinning, growth retardation, relapse, and adrenal gland suppression (8). Almost half of the atopic eczema patients use complementary and alternative therapies, such as traditional Chinese medicine (TCM) (9). TCM may be a supplement or replacement for western medicine for the long-term treatment of eczema.

In China, patients with atopic dermatitis often receive alternative and complementary treatments as firstline therapy. The herbal medicines used to prevent the development and recurrence of atopic dermatitis are widely accepted. However, to date, only a few studies have provided conclusive evidence regarding the efficacy of oral ingestion or topical application of Chinese herbal medicines (CHM) in relation to adverse events (AEs) compared with other control interventions $(10,11)$, due to methodological weaknesses of randomized controlled trials (RCTs). Only a few RCTs have demonstrated the efficacy (or lack of efficacy) of Chinese medicinal herbs in treating atopic eczema. Further, larger scale RCTs in this direction are warranted (12). Some researchers have reported the benefits of Runzaozhiyang capsules (RZZYC, component as shown in Table 1) in alleviating the symptoms of eczema (13).

Qinzhuliangxue decoction (QZLXD), an effective formula developed in the 1950s by Xia's Chinese medical surgery (one of the most famous Shanghai styles of TCM) for atopic dermatitis has been clinically used for over 50 years. It comprises six Chinese herbs, as shown in 
Table 2, and has been approved by the Shanghai Food and Drug Administration (Petition Number: YZ170063). Previous studies have reported that QZLXD had satisfactory effects in alleviating symptoms of atopic eczema and maintaining long-term stability, thus improving patient quality of life (14). Although positive outcomes associated with QZLXD have been clinically observed, strong evidence is lacking. Therefore, we conducted an RCT on QZLXD to investigate its safety, clinical efficacy, and influence on adult quality of life in the treatment of atopic dermatitis in comparison with a placebo treatment.

\section{Methods}

\section{Participants}

Patients were selected based on the following inclusion and exclusion criteria. Inclusion criteria: (I) a diagnosis of subacute atopic eczema (15); (II) aged between 18 and 65 years; (III) affected body surface area between $3 \%$ and $10 \%$ (inclusive) as assessed by the palm method; (IV) skin lesions on the trunk or extremities, excluding the palms/soles, face/scalp, and vulvar areas; $(\mathrm{V})$ willingness for participation and follow-up with a readiness to sign informed consent.

Exclusion criteria: (I) allergies to any medicine or ingredients used in this study; (II) pregnancy, lactating, or planning a pregnancy within a year; (III) clinically meaningful laboratory findings that could affect patient safety; (IV) history of primary cardiovascular, respiratory, digestive, urinary, endocrinological, and hematological diseases that cannot be controlled with ordinary treatments; (V) topical or systematic application of corticosteroids within the previous 1 and 2 weeks, respectively; (VI) participation in another clinical trial within the previous 3 weeks; (VII) non-cooperation, including language barrier and inability to reach the Research Center for medical treatment or other conditions or diseases that the researchers believe may cause significant risks or obscure the research findings.

\section{Sample size}

Based on the difference between two sample rates, using the estimation formula to calculate the required sample size (16). The total effective rate at the end of treatment was defined as the effect index, and the predicted effective rates were $86.3 \%$ and $75.0 \%$ for the treatment and control groups, respectively (17). The inspection level $(\alpha)$ was 0.05 and power was $0.8(\beta=1$; power $=0.2)$ were assumed. Based on the two-sided tests, the sample size was estimated to be 146, with 73 subjects per group. Owing to possible patient withdrawal, we increased the sample size by $20 \%$, and a total of 176 subjects were required, with 88 participants in each group.

\section{Study design}

The randomization sequence was generated by study investigators who are statisticians. To balance the baseline characteristics between the two groups, subjects were assigned in a 1:1 ratio. The enrolled subjects were randomly assigned to receive $30 \mathrm{~mL}$ of QZLXD ( $\mathrm{n}=82)$ twice daily after meals, or 4 tablets of RZZYC three times a day $(n=86)$ after meals and an additional daily mizolastine sustainedrelease tablet. Eligible patients were randomly assigned to one of the two groups on the second.

The dosage used in the present study was determined according to the Chinese Pharmacopoeia (2010 edition). Quality control of the product was performed by detecting baicalin and paeonol with high-performance liquid chromatography (HPLC), as shown in Figure 1.

\section{Ethics}

The study protocol was approved by the institutional review boards (IRBs) of Yueyang Integrated Traditional Chinese and Western Medicine Hospital, independent National Ethics Committee (EC), and Chinese Medicinal Agency (Approval Number: 2016-017). All participants provided their written informed consent before enrollment.

\section{Study outcomes}

The primary outcome was the Eczema Area and Severity Index (EASI) score achieved with treatment. The secondary outcome was quality of life. EASI, Dermatology Life Quality Index (DLQI), and itching scores were measured before treatment and during weeks 0,2 , and 4 . The condition of all participants improved after 4 weeks of treatment, and they were monitored until disease relapse or until 8 weeks. The recurrent time as well as the EASI, DLQI, and itching scores after relapse were calculated at the week 8 visits if relapse occurred. The flow chart of the study is shown in Figure 2.

The primary efficacy endpoint for each group was the change in EASI score after 4 weeks of treatment compared with the baseline value. EASI 95, 60, 30 and $\leq 30$ corresponded to EASI $\geq 95 \%$ (cured), between $60 \%$ 
A

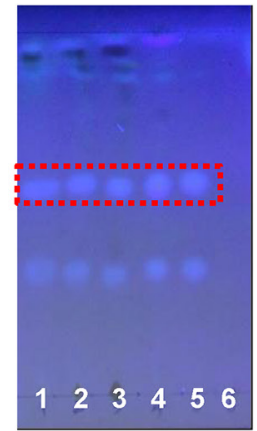

Saposhnikovia divaricata

B
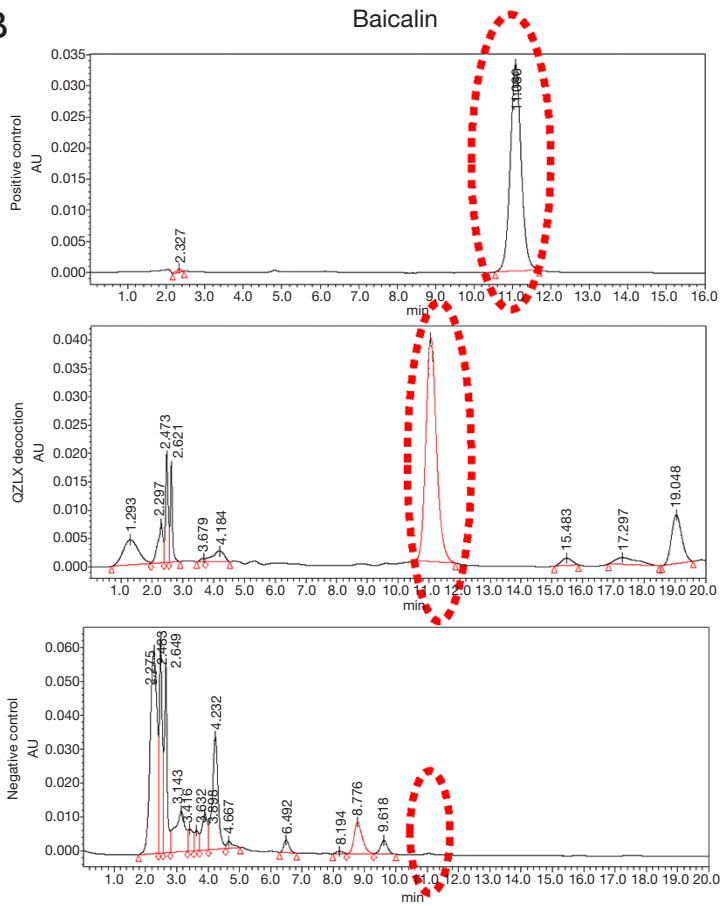

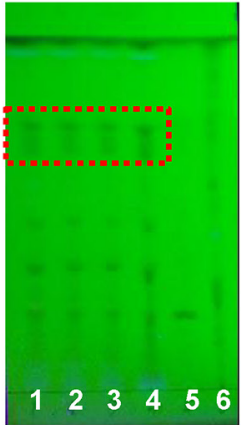

Liquorice

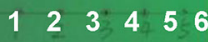

Cortex Moutan

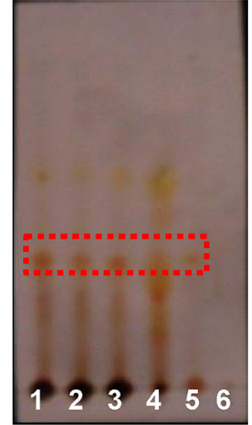

Scutellaria baicalensis

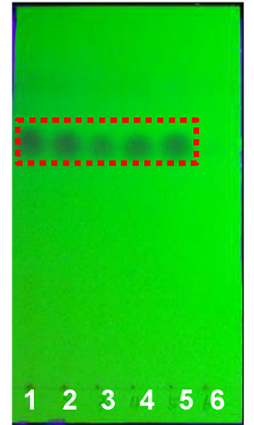

Coix seed
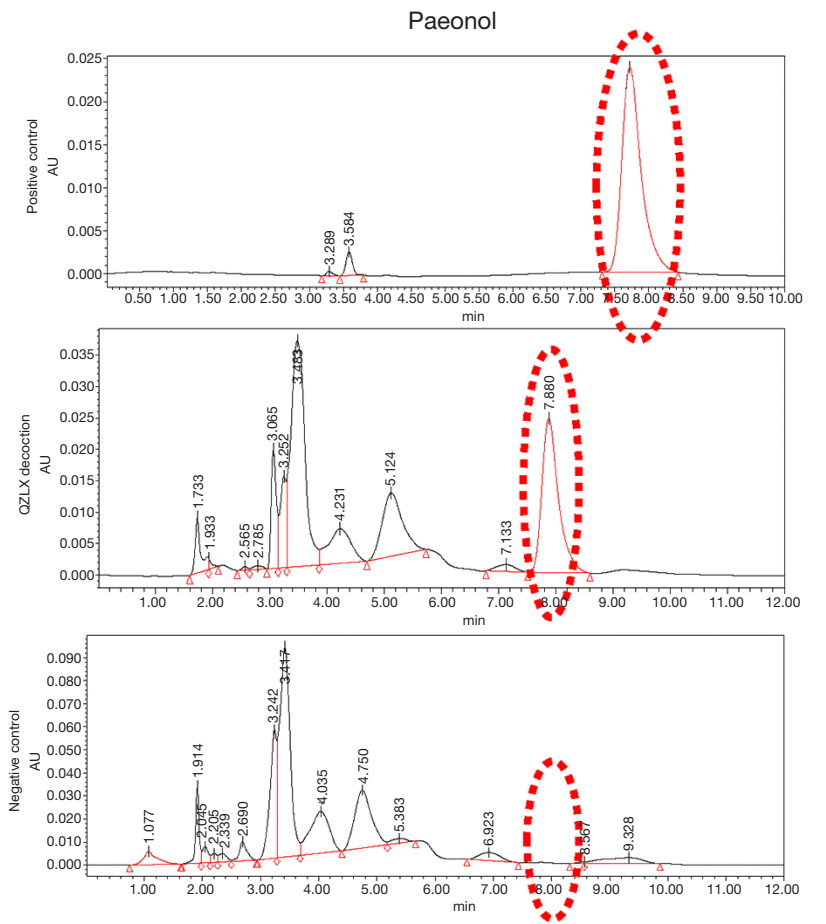

Figure 1 The qualitative and quantitative analysis of QZLX products. (A) Different herbs and three different batches of QZLX products were quantified using thin-layer chromatography. Samples analyzed per well: [1], [2], and [3] are test samples, [4] controlled medicinal herbs, [5] controlled drugs, and [6] negative control. (B) Quality control of QZLXD by the HPLC method. The expression levels of baicalin and paeonol measured by HPLC were used as quality controls for the obtained QZLX product. Baicalin acid and paeonol were detected in the positive control and QZLX samples, but not in the negative control (B). The dashed box means QZLX products have the same positive result with control; the dashed oval means QZLX product has the same result with positive control. QZLX, Qinzhuliangxue; QZLXD, Qinzhuliangxue decoction; HPLC, high-performance liquid chromatography.

and $94 \%$ (significantly effective), between $30 \%$ and $59 \%$ (effective), and $<30 \%$ (invalid), respectively.

Three secondary endpoints were considered: (I) DLQI and itching scores at week 2 compared with the baseline scores; (II) decreased rate of DLQI and itching scores at every visit compared to those at baseline; (III) relapse rate at week 8 post-treatment. Recurrence was defined as an EASI score $\geq 10 \%$ of the EASI baseline; relapse rate $=$ recurrent patients/patients of followed up $\times 100 \%$.

\section{Safety}

Safety evaluation was performed by: (I) blood routine tests for red blood cell, white blood cell, and platelet counts, 


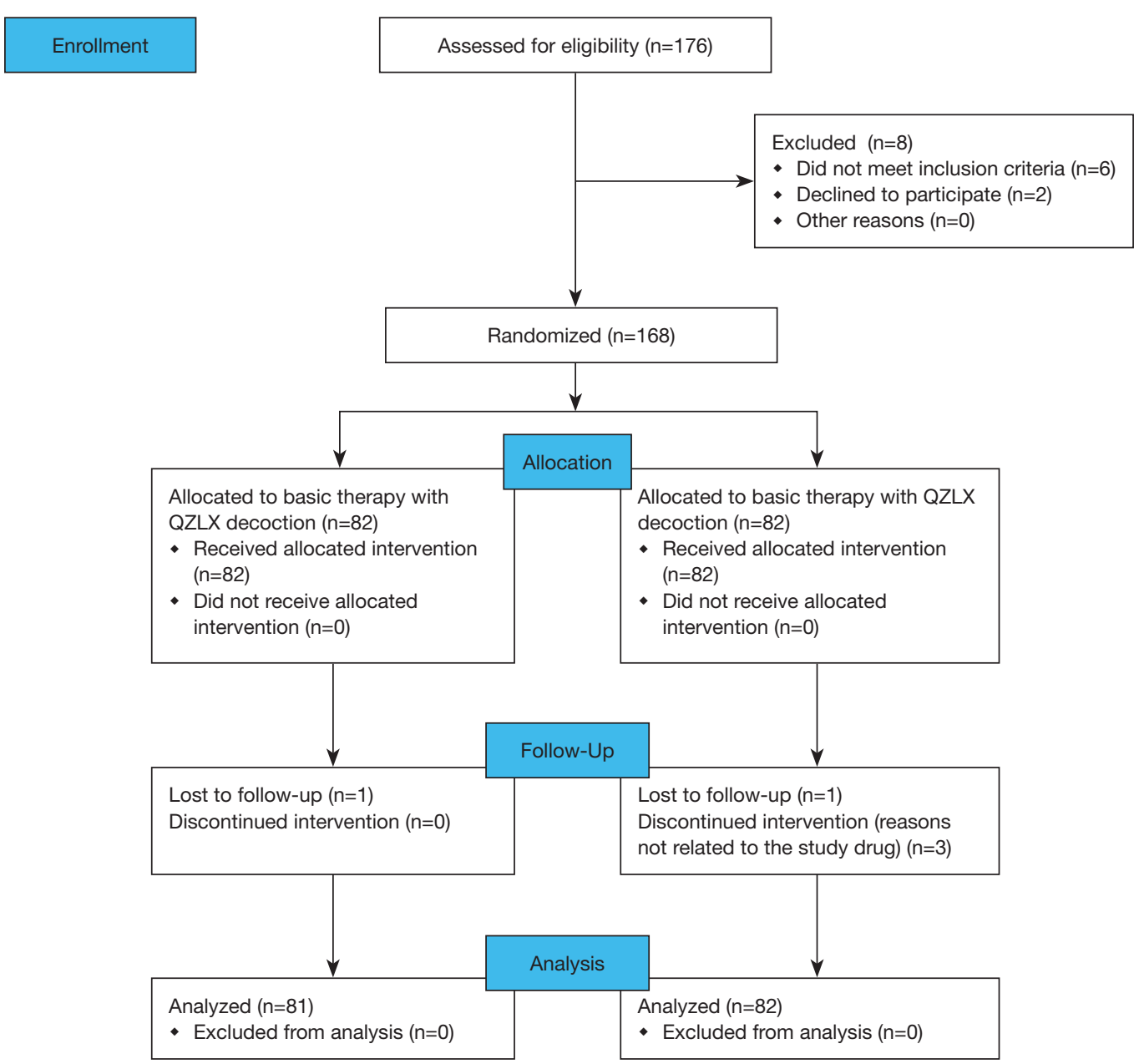

Figure 2 Flowchart of the trial, enrollment, randomization, treatment, follow-up, and data analysis.

(II) urine routine tests for red blood cell and white blood cell counts, and of protein and glucose levels, (III) hepatic function tests for glutamic pyruvic transaminase, glutamic oxalacetic transaminase, total bilirubin, and glutamyl transpeptidase levels, and (IV) renal function tests for blood urea nitrogen and creatinine levels.

\section{Statistical analysis}

In this study, quantitative variables (EASI, itch score, and DLQI) with normal distribution were described as means and standard deviation (SD); non-normal distribution were summarized as median and interquartile range (IQR). Comparative analysis with baseline values was performed by $\mathrm{F}$ test for quantitative data such age, sex and duration of the disease and the Mann-Whitney test for categorical data, such as itching score. The comparison of AEs between the two groups was assessed by Fisher exact probability method. The measurer was blinded to the results.

\section{Results}

\section{Comparison of baseline characteristics between patients}

Patients were recruited from July 2014 to the end of September 2016. A total of 168 individuals were screened at the Yueyang Integrated Traditional Chinese and Western Medicine Hospital for eligibility (Figure 2). At the end of the trial, 163 out of 168 patients completed the trial with a dropout rate of $3.0 \%$ (Figure 2). Of the 5 patients who dropped out, 2 were dissatisfied with the treatment process, 2 could not continue because of their work schedule, and 1 
failed to complete follow-up. The baseline characteristics of patients are reported in Table 3. No significant differences in the following variables were observed: age, mean of course of the disease, and EASI, DLQI, and itching scores.

The mean age of the treatment group was $44.81 \pm$ 15.00 years $(n=82) ; 60.49 \%$ of the patients were men. The mean age of the control group was $48.16 \pm 13.56$ years $(n=86)$; $46.34 \%$ of the patients were men. The mean of EASI scores were $14.17 \pm 6.90$ for the treatment group and $14.17 \pm 9.84$ for the control group. The mean DLQI scores were $11.38 \pm 6.40$ and $9.80 \pm 5.91$ for the treatment and control groups, respectively. The median itching score in both groups was 2.00 (range, 0.00 to 3.00 ).

\section{Efficacy}

\section{Primary outcome}

The EASI score after 4 weeks of treatment was compared with the score at baseline. The mean EASI scores after 4 weeks of treatment were $3.24 \pm 2.91$ in the treatment group and $4.99 \pm 4.16$ in the control group. A significantly higher proportion of patients receiving QZLXD achieved higher total effective rate after treatment than those receiving RZZYC ( $\mathrm{P}=0.003)$. The total effective rate $(\%, 95 \% \mathrm{CI})$ was $77.78(68.53,87.03)$ in the QZLXD group and 64.63 $(54.06,75.20)$ in the control group (Tables 4,5$)$.

\section{Secondary outcomes}

\section{EASI score after treatment}

There was no difference in the EASI scores between the QZLXD and the control groups $(8.75 \pm 4.09$ versus $9.02 \pm 6.00, \mathrm{P}>0.05)$ after a 2 weeks of treatment; however, a difference in EASI score was observed after 4 weeks of treatment (Table 5 and Figure 3), indicating that QZLXD required a certain amount of time to elicit therapeutic effects. The conditions of QZLXD-treated patients markedly improved after treatment, with a decrease in erythema, dispel swelling, and pruritus (Figure 4).

\section{DLQI score after treatment}

After 4 weeks of treatment, the mean DLQI was $3.11 \pm 2.69$

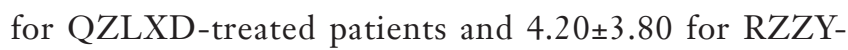
treated patients. However, the difference was not statistically significant at week 4 of treatment $(\mathrm{P}=0.053)$ or at every visit during follow-up $(\mathrm{P}>0.05)$ (Table 5).

In addition, QZLXD treatment had a positive effect on the itching score. The mean itching score after 2 weeks was $1.26 \pm 0.9$ in the treatment group and $1.96 \pm 0.48$ in the control group. However, after 4 weeks, the itching score was $0.93 \pm 0.61$ in the treatment group and $1.24 \pm 0.51$ in the control group. Furthermore, significantly higher proportions of QZLXD-treated patients achieved a higher itching score than those receiving RZZY at the 2-week and 4-week visits $(\mathrm{P}=0.001)$ (Table 5).

\section{Recurrence}

The recurrent rates $(\%, \mathrm{n} / \mathrm{N})$ were $6.17(5 / 81)$ in the QZLXD group and 20.73 (17/82) in the control group. There was also a significant difference between the two groups in terms of recurrence rate $(\mathrm{P}=0.007)$, suggesting that subjects in the QZLXD group experienced lesser recurrence than those in the control group, consistent with the trend of effective ratio (Table 4).

\section{Safety}

Safety was evaluated based on the occurrence of AEs. A total of 16 AEs were reported: 6 in the treatment group and 10 in the control group. All the AEs occurred during the treatment period (within 4 weeks). The overall comparison of $\mathrm{AE}$ frequencies between the two groups was not significantly different $(\mathrm{P}=0.434)$ (Table 6).

\section{Discussion}

In the last 20 years, QZLXD has been extensively used for treating eczema, as its clinical efficacy in terms of relieving symptoms and reducing the frequency of relapse of eczema has been established (18). QZLXD also has anti-inflammatory effects, enhancing the itching threshold induced by histamine phosphate, which could also be used for treating allergies. The present randomized, controlled trial explored the benefits of administering QZLXD compared with those of conventional treatment for eczema, showing a superior efficacy after 4 weeks of treatment.

Herbal remedies have been traditionally used to control dermatitis, including Indian pennywort, walnut, and turmeric (19). TCM in the form of topical agents such as creams are also popular for treating dermatitis (20-22). Owing to the fewer side effects, TCM has drawn wide and international attention in treating eczema (23). The National Health Insurance Research Database in Taiwan identified the largest number of patients with winddampness-heat pattern (24), which could be treated with 
Table 3 Baseline characteristics of the participants

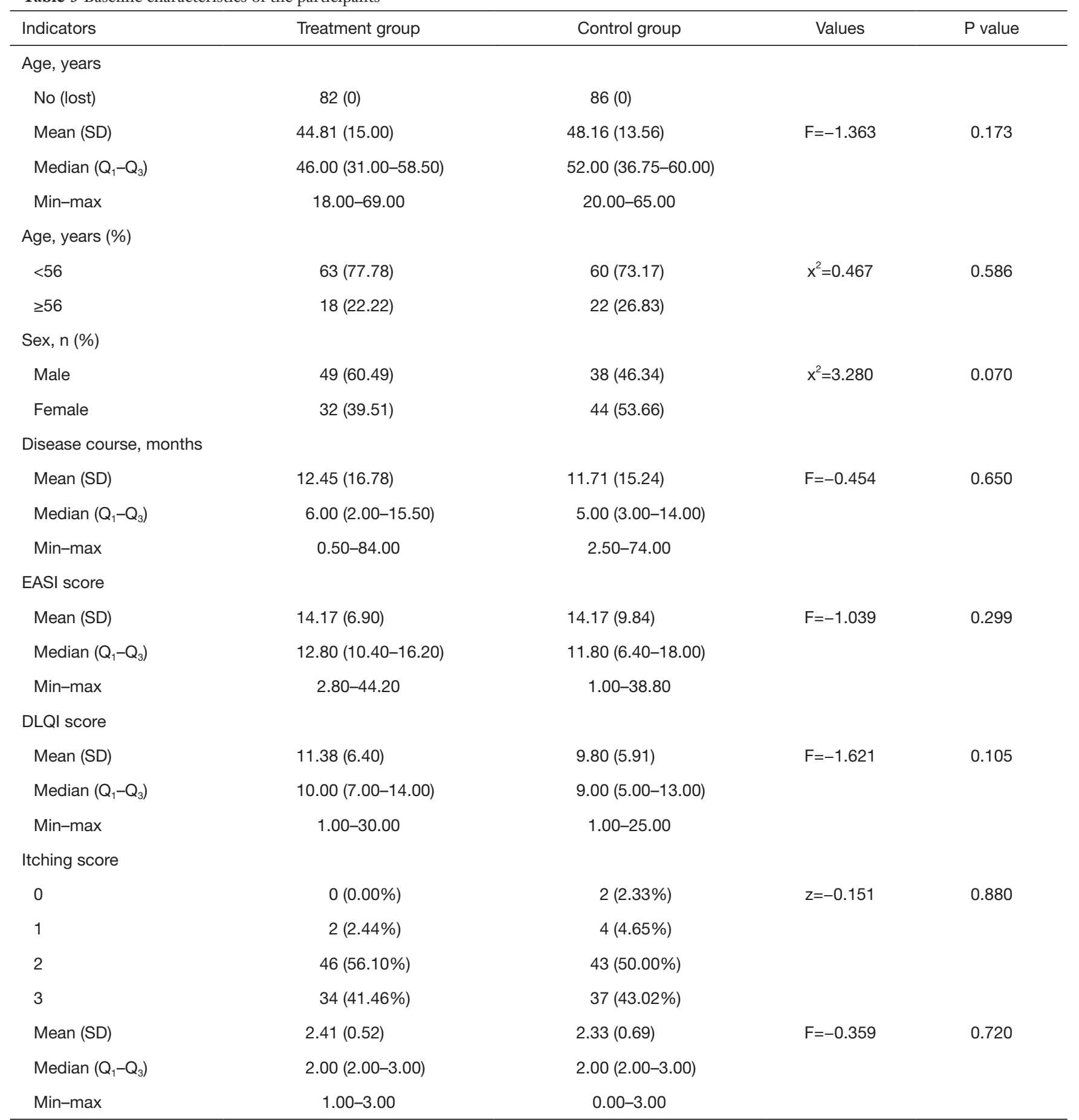

Comparative analysis with baseline values was performed by $\mathrm{F}$ test for quantitative data such age, sex and duration of the disease and the Mann-Whitney test for categorical data, such as itching score. 
Table 4 Comparison of primary outcome and recurrent rate between both groups

\begin{tabular}{|c|c|c|c|c|c|}
\hline Time point & EASI & Treatment group $(n=81)$ & Control group $(n=82)$ & $\chi^{2}$ & $P$ value \\
\hline \multirow{3}{*}{ Week 2} & EASI 60 & 1 & 3 & & \\
\hline & EASI 30 & 62 & 51 & & \\
\hline & Invalid & 18 & 28 & & \\
\hline \multirow[t]{4}{*}{ Week 4} & EASI 95 & 24 & 6 & & \\
\hline & EASI 60 & 39 & 47 & & \\
\hline & EASI 30 & 16 & 25 & & \\
\hline & Invalid & 2 & 4 & & \\
\hline
\end{tabular}

The primary outcome between the two groups was compared by $\chi^{2}$ test. The Fisher exact probability method was used to compare the recurrent rate between groups. EASI decrease rate = (EASI baseline score - EASI after treatment)/EASI baseline $\times 100 \%$. The criteria for efficacy were as follows: (I) EASI 95, EASI decrease rate $\geq 95 \%$; (II) EASI 60, EASI decrease rate between 60-94\%; (III) EASI 30, EASI decrease rate between 30-59\%; and (IV) invalid, EASI decrease rate $<30 \%$. Q1-Q3: inter quartile range. Total effective rate = (EASI 95 number + EASI 60 number)/number $\times 100 \%$. Recurrence was defined as EASI score $\geq 10 \%$ of EASI baseline, recurrent rate $=$ recurrent patients/patients of followed up $\times 100 \%$.

Table 5 Comparison of EASI, DLQI, and itching scores between both groups before and after treatment

\begin{tabular}{|c|c|c|c|c|c|c|}
\hline Variable & Time point & Number & Treatment group $(\mathrm{n}=82)$ & Control group $(n=86)$ & F \& z (inter-group) & $P$ value \\
\hline \multirow{8}{*}{ EASI } & \multirow{3}{*}{ Baseline } & Mean (SD) & $14.17(6.90)$ & $14.17(9.84)$ & \multirow{3}{*}{$F=-1.039$} & \multirow{3}{*}{0.299} \\
\hline & & Median $\left(Q_{1}-Q_{3}\right)$ & $12.80(10.40-16.20)$ & $11.80(6.40-18.00)$ & & \\
\hline & & Min-max & $2.80-44.20$ & $1.00-38.80$ & & \\
\hline & \multirow{3}{*}{ Week 2} & Mean (SD) & $8.75(4.09)$ & $9.02(6.00)$ & \multirow{3}{*}{$F=-0.420$} & \multirow{3}{*}{0.675} \\
\hline & & Median $\left(Q_{1}-Q_{3}\right)$ & $8.00(6.50-10.60)$ & $8.60(4.20-12.10)$ & & \\
\hline & & Min-max & $1.80-30.20$ & $0.60-26.00$ & & \\
\hline & \multirow[t]{2}{*}{ Week 4} & $\mathrm{~N}[\mathrm{~N}$ miss $]$ & $82[1]$ & $86[4]$ & \multirow[t]{2}{*}{$F=-2.965$} & \multirow[t]{2}{*}{0.003} \\
\hline & & Min-max & $0.00-15.20$ & $0.40-19.20$ & & \\
\hline \multirow[t]{4}{*}{ DLQI score } & \multirow[t]{4}{*}{ Baseline } & $\mathrm{N}[\mathrm{N}$ miss $]$ & $82[0]$ & $86[0]$ & \multirow[t]{4}{*}{$F=-1.621$} & \multirow[t]{4}{*}{0.105} \\
\hline & & Mean (SD) & $11.38(6.40)$ & $9.80(5.91)$ & & \\
\hline & & Median $\left(Q_{1}-Q_{3}\right)$ & $10.00(7.00-14.00)$ & $9.00(5.00-13.00)$ & & \\
\hline & & Min-max & $1.00-30.00$ & $1.00-25.00$ & & \\
\hline
\end{tabular}

Table 5 (continued) 
Table 5 (continued)

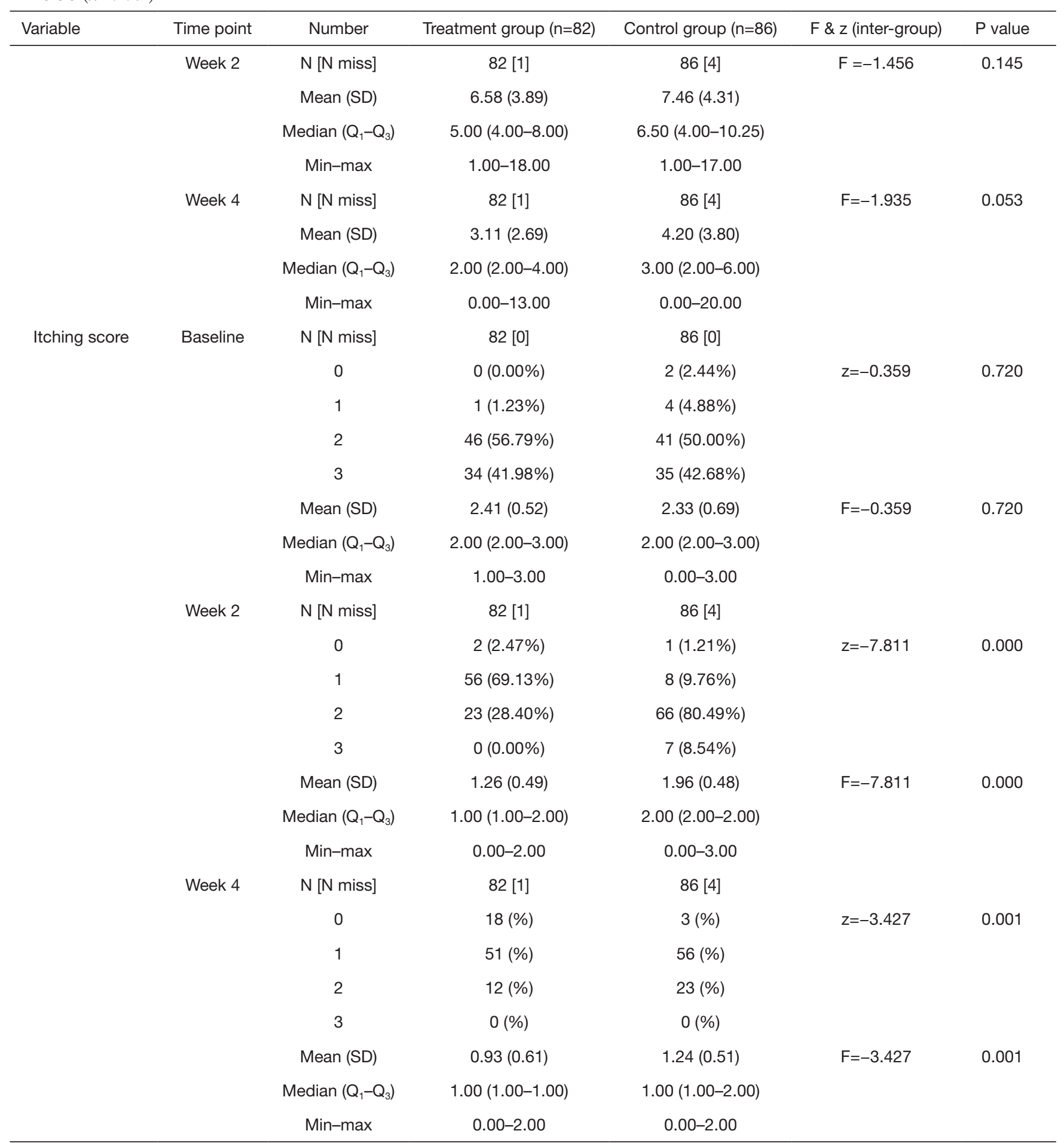

Itching score was measured by the Mann-Whitney test for grading data and the F test was used for other comparative analyses. Q1-Q3: inter quartile range. $\mathrm{N}$ miss: number of missing values. 
A

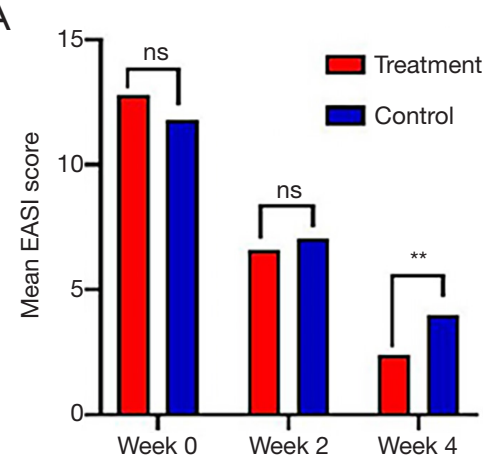

B

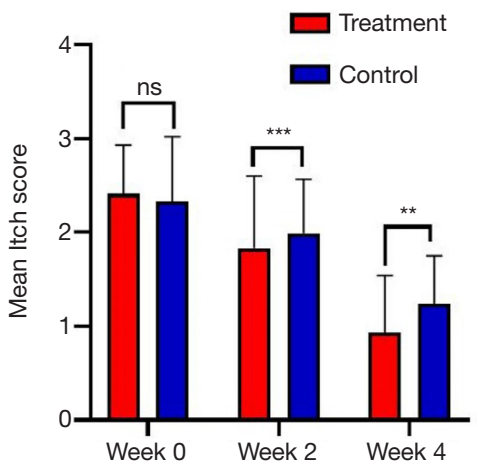

C

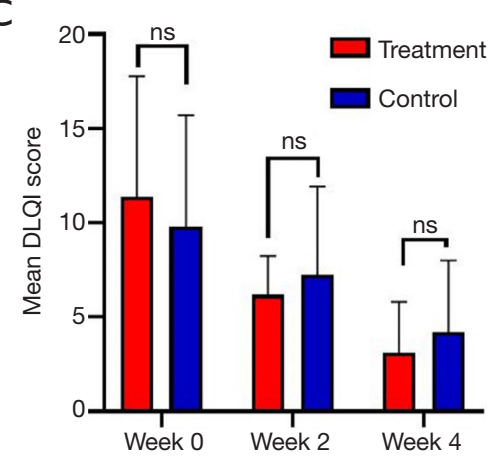

Figure 3 Comparison of the evaluation index between the groups. (A) EASI score. (B) Itching score. (C) DLQI score. ${ }^{* *}, \mathrm{P}<0.01,{ }^{* * *}$, $\mathrm{P}<0.001$ vs. control. EASI: Eczema Area and Severity Index; DLQI, Dermatology Life Quality Index.
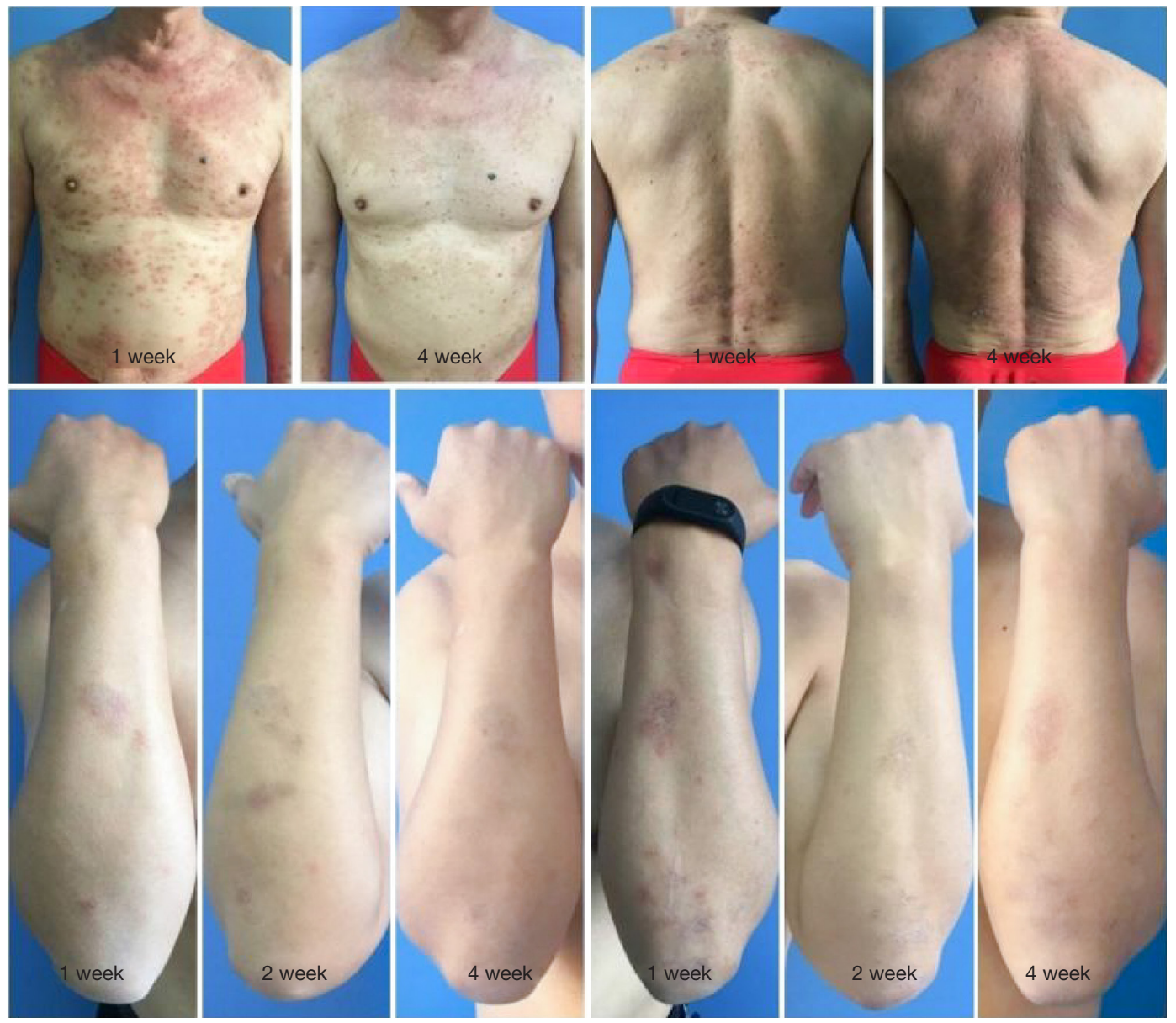

Figure 4 Condition of QZLXD-treated patients markedly improved after the treatment, effectively reducing erythematic, dispel swelling, and antipruritic effects. QZLXD, Qinzhuliangxue decoction. 
Table 6 Adverse events in both groups

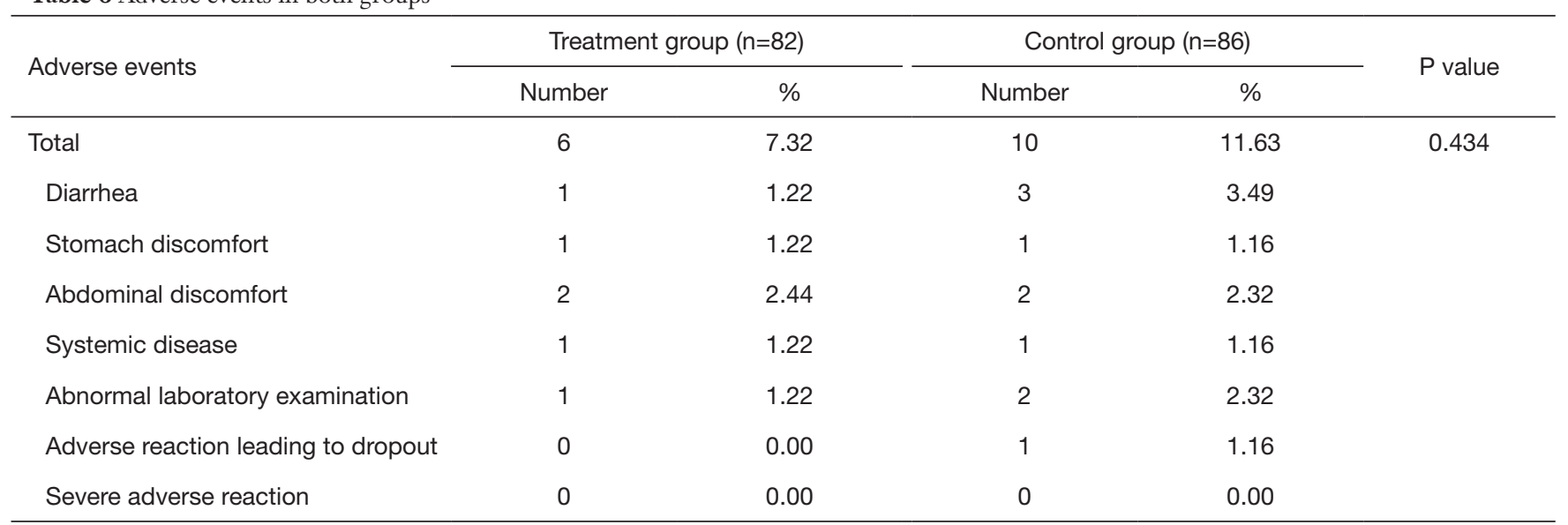

The comparison of adverse events between the two groups was assessed by Fisher exact probability method.

TCM. Despite this, various Chinese medicine educational institutions have different views on this topic.

Xia's (25) Chinese medical surgery suggested the improvement theory that "blood-heat can be used as the basic syndrome type, which is the cause of the disease, and Yang float is the symptom of the disease complicated with dampness pattern and wind evil". The theories that "expelling nearby pathogens as early as possible protects the yin fluid, and that blood heat induces yang floating" were demonstrated using Scutellaria baicalensis, mother-ofpearl, peony bark, Lithospermum, Saposhnikovia, oyster, and magnetite, with good clinical efficacy (25).

Huang Qin (Scutellaria baicalensis) can remove heat, dampness, and toxicity especially in Middle-Jiao (26), with anti-inflammatory, antioxidant, anticancer, and immuneregulatory functions (27). In addition, large doses of Huang Qin (Scutellaria baicalensis) and Zhen Zhumu (mother-ofpearl), the major components of QZLXD, are of great importance to achieve the therapeutic and combined effects of eliminating dampness and heat via enhancing the antiinflammatory and antiallergic effects. According to the theory of Xia, occurrence of skin diseases was closely linked to heat, either virtual or actual heat, and the syndrome of dampness retention has a heat-cleaning effect by eliminating wind dampness and heat through itching (28). Another important use of the decoction is in heavy material therapy, also known as mind-tranquilizing therapy, in TCM; the decoction includes Zhen Zhumu (mother-of-pearl), Mu Li (oysters), Ci Shi (magnetite), and other therapeutic agents. These heavy materials consist mainly of ore, fossil, or testaceous drugs, which are used to treat irritability, insomnia, and panic epilepsy (29,30). Through long-term clinical observation, the theory of "Suppressing Hyperactive Liver-Yang and Strong Tranquilizing Effect" is the principle of our department to treat eczema.

In the present study, some patients had a history of bronchial asthma, which is consistent with observations of previous studies, mainly the frequent association of eczema with other atopic disorders, such as asthma and high fever (31). Thus, even environmental factors may play an important role in eczema treatment (32). Besides, some evidence suggests that urbanization also increases the incidence of eczema (33). The cause of eczema remains unknown, but the recurrence rate suggests a multifactorial cause, including genetic (34), environmental, and socioeconomic factors (35). Those contributing factors may interact in various ways that ultimately lead to epidermal barrier impairment, unchecked type 2 immunity, and chronic disease (36). QZLXD can enhance both innate and adaptive immunity despite the complicated pathogenesis of eczema. However, one daily mizolastine sustained-release tablet and the use of external drugs are needed. Maintaining skin moisturization and using topical corticosteroids and/ or calcineurin inhibitors are still the most basic approach for treating the disease. Anti-inflammatory treatment may also be necessary (37). The decision to use these therapies should be based on efficacy and safety readouts from well designed, long-term trials (38).

Some limitations impacted the present study: (I) there were difficulties in recruiting patients to the QZLXD group. This may have been due to hesitation to take the decoction. Therefore, the development of safe and effective 
granules may be a better option for the treatment and prevention of eczema with this drug. (II) Even though the trial adherence rate was acceptable (95.5\%), increasing the sample size, which likely enhances the statistical significance of the results, and increasing the treatment and follow-up periods are recommended. Considering that eczema is a common lingering skin disease that needs a long course of treatment, a 4-week treatment period is too short to provide an accurate assessment of the long-term efficacy and safety of QZLXD. (III) It is unclear from this study whether other formulations of the drug (such as a cream or other) or a greater intervention intensity may provide further benefits.

\section{Conclusions}

In conclusion, this study showed that QZLXD was potentially safe and more effective than RZZYC in treating sub-acute eczema of blood-heat type. Our results suggest that QZLXD is effective in relieving symptoms of eczema, reducing relapse rates, and enhancing the itching threshold of histamine phosphate.

\section{Acknowledgments}

Funding: Sponsored by Shanghai Sailing Program (Grant No. 17YF1419900). NSFC of China (Grant No. 81874470), National Key Research and Development Program of China (Grant No. 2018YFC1705301), Shanghai Science and Technology Committee (Grant No. 14401972703), Shanghai Development Office of TCM [Grant No. ZY (2018-2020)-FWTX-1008, ZY (2018-2020)-CCCX-200408, ZY (2018-2020)-FWTX-4010].

\section{Footnote}

Conflicts of Interest: All authors have completed the ICMJE uniform disclosure form (http://dx.doi.org/10.21037/ apm.2020.04.17). The authors have no conflicts of interest to declare.

Ethical Statement: The authors are accountable for all aspects of the work in ensuring that questions related to the accuracy or integrity of any part of the work are appropriately investigated and resolved. The study protocol was approved by the institutional review boards (IRBs) of Yueyang Integrated Traditional Chinese and Western Medicine Hospital, independent National Ethics Committee (EC), and Chinese Medicinal Agency (Approval
Number: 2016-017). All participants provided their written informed consent before enrollment.

Open Access Statement: This is an Open Access article distributed in accordance with the Creative Commons Attribution-NonCommercial-NoDerivs 4.0 International License (CC BY-NC-ND 4.0), which permits the noncommercial replication and distribution of the article with the strict proviso that no changes or edits are made and the original work is properly cited (including links to both the formal publication through the relevant DOI and the license). See: https://creativecommons.org/licenses/by-nc-nd/4.0/.

\section{References}

1. Rożalski M, Rudnicka L, Samochocki Z. Atopic and Nonatopic Eczema. Acta Dermatovenerologica Croatica 2016;24:110-5.

2. Eichenfield LF, Tom WL, Chamlin SL, et al. Guidelines of care for the management of atopic dermatitis: section 1 . Diagnosis and assessment of atopic dermatitis. J Am Acad Dermatol 2014;70:338-51.

3. Wang X, Shi XD, Li LF, et al. Prevalence and clinical features of adult atopic dermatitis in tertiary hospitals of China. Medicine 2017;96:e6317.

4. Hua T, Silverberg JI. Atopic dermatitis in US adults: Epidemiology, association with marital status, and atopy. Ann Allergy Asthma Immunol 2018;121:622-4.

5. Drucker AM, Wang AR, Li WQ, et al. The Burden of Atopic Dermatitis: Summary of a Report for the National Eczema Association. J Invest Dermatol 2017;137:26-30.

6. Leung TN, Hon KL. Eczema therapeutics in children: What do the clinical trials say? Hong Kong Med J 2015;21:251-60.

7. Eichenfield LF, Tom WL, Berger TG, et al. Guidelines of care for the management of atopic dermatitis. Section 2. Management and treatment of atopic dermatitis with topical therapies. J Am Acad Dermatol 2014;71:116-32.

8. Sidbury R, Davis DM, Cohen DE, et al. American Academy of Dermatology Guidelines of care for the management of atopic dermatitis. Section 3. Management and treatment with phototherapy and systemic agents. J Am Acad Dermatol 2014;71:327-49.

9. Gu S, Pang C, Xue CC, et al. Chinese herbal medicine for atopic eczema. The Cochrane Library, 2010.

10. Gu S, Yang AW, Li CG, et al. Topical application of Chinese herbal medicine for atopic eczema: A systematic review with a meta-analysis. Dermatology 2014;228:294-302. 
11. Gu S, Yang AW, Xue CC, et al. Chinese herbal medicine for atopic eczema. Cochrane Database Syst Rev 2013;(9):CD008642.

12. Hon KL, Chan CL, Leung PC. Chinese herbal medicine research in eczema treatment. Chin Med 2011;6:17.

13. Huang D, Chen K, Zhang FR, et al. Efficacy and safety of Run Zao Zhi Yang capsule on chronic eczema: a multiplecenter, randomized, double-blind, placebo-controlled clinical study. J Dermatolog Treat 2019;30:677-84.

14. Li S, Chen J, Li B, et al. Clinically randomized controlled trials of QZLXD for atopic dermatitis. World Clin Drugs 2016;37:173-6.

15. Wasserbauer N, Ballow M. Atopic Dermatitis. Am J Med 2009; 122:121-5.

16. Deng W, He J. Design and statistically analysis of clinical trials. Beijing: People's Medical Publishing House, 2012.

17. Wuquan H. Clinical study on Runzaozhiyang capsule combined with mizolastine to treat chronic eczema. China Foreign Medical Treatment 2011;30:107.

18. Fan B, Li B, Jin R, et al. Anti-inflammatory and Relieving Itching of Qinzhu Liangxue Mixture and Its Experimental Study. Chinese Journal of Dermatovenereology of Integrated Traditional \& Western Medicine, 2008.

19. Khiljee S, Rehman N, Khiljee T, et al. Formulation and clinical evaluation of topical dosage forms of Indian Penny Wort, walnut and turmeric in eczema. Pak J Pharm Sci 2015;28:2001-7.

20. Liu L, Ying L, Mi Z, et al. Tripterygium agents for the treatment of atopic eczema: A Bayesian analysis of randomized controlled trials. Phytomedicine 2019;59:152914.

21. Xu R, Li FL, Zhang LL, et al. Herba Saxifragae cream in treatment of chronic eczema: a randomized controlled trial. Zhong Xi Yi Jie He Xue Bao 2008;6:1246-9.

22. Hon KL, Lo W, Cheng WK, et al. Prospective selfcontrolled trial of the efficacy and tolerability of a herbal syrup for young children with eczema. J Dermatolog Treat 2012;23:116-21.

23. Zhi YL. Pharmacodynamics and clinical research progress on anti-inflammatory effect of licorice. Guangming Journal of Chinese Medicine 2017;19:2895-8.

24. Chen HY, Lin YH, Hu S, et al. Identifying Chinese herbal medicine network for eczema: implications from a nationwide prescription database. Evid Based Complement Alternat Med 2015;2015:347164.

25. Hua L, Zhang M, Zhou M. Brief discussion on the academic influence of theory of epidemic febrile diseases on Xia's Chinese medical surgery. Shanghai Journal of Traditional Chinese Medicine 2017;51:12-4.

26. He K. TCM Treatment for Two Cases of Chronic and Intractable Eczema. J Tradit Chin Med 2008;28:98-100.

27. Chi GF, Ding L, Chang LM, et al. The Present Domestic Pharmacological Advance on Scutellaria Baicalensis. J Inner Mongolia Univ Nationalities (Natural Sciences) 2005;20:207-9.

28. Li JY, Zhang Li. The application of Zhongzhen medicine in dermatology. $\mathrm{C}$ hint $\mathrm{J}$ Dermato $\mathrm{V}$ enerol Integ Trad $\mathrm{W}$ Med 2012;11:324-5.

29. Li S, Li X, Li B. Blood heat syndrome of psoriasis vulgaris treated with Jing's experience. Journal of Yunnan College of Traditional Chinese Medicine 2014;37:88-9.

30. Wu L, Liu S, Wu D, et al. Research progress and clinical application of traditional Chinese mineral medicine pharmacological effects of soothing the nerves. Modern Chinese Medicine 2015;9:892-8.

31. Williams HC, Wüthrich B. The natural history of atopic dermatitis. In: Williams HC. editor. Atopic Dermatitis. Cambridge University Press, 2000;49-59.

32. Wang IJ, Guo YL, Weng HJ, et al. Environmental risk factors for early infantile atopic dermatitis. Pediatr Allergy Immunol 2007;18:441-7.

33. Grover S, Grewal RS. Fitzpatrick's Dermatology in General Medicine 2015;150:794.

34. Friedmann PS. The pathogenesis of atopic eczema. Hospital Medicine 2002;63:653-6.

35. McNally NJ, Williams HC, Phillips DR. Atopic eczema and the home environment. Br J Dermatol 2001;145:730-6.

36. Eyerich K, Eyerich S, Biedermann T. The Multi-Modal Immune Pathogenesis of Atopic Eczema. Trends Immunol 2015;36:788-801.

37. Kawakami T, Soma Y. Correlation of livedo racemosa, cutaneous inflammatory plaques, and antiphospholipid antibodies in patients with cutaneous polyarteritis nodosa. Medicine 2011;90:119-24.

38. Prezzano JC, Beck LA. Long-Term treatment of atopic dermatitis. Dermatol Clin 2017;35:335-49.

Cite this article as: Ma T, Chai $\mathrm{Y}, \mathrm{Li} \mathrm{S}$, Sun $\mathrm{X}$, Wang $\mathrm{Y}, \mathrm{Xu}$ R, Chen J, Zhou M, Zhou M, Li B, Xu W, Li X. Efficacy and safety of Qinzhuliangxue decoction for treating atopic eczema: a randomized controlled trial. Ann Palliat Med 2020;9(3):870-882. doi: 10.21037/apm.2020.04.17 